\title{
Retos de los stakeholders del ámbito de la discapacidad en materia de empleo tras la pandemia de COVID-19
}

\section{Stakeholder challenges in the employment disability's sector after the COVID-19 pandemic}

\section{Palabras clave}

Discapacidad, empleo, COVID-19, efectos, necesidades sociales, retos, empleabilidad.

\section{Keywords}

Disability, employment, COVID-19, effects, social needs, challenges, employability.

\section{Elena Ortega Alonso \\ <eortega@siis.net>}

Centro Español de Documentación sobre Discapacidad (CEDD). España

\section{El impacto del COVID-19}

El 13 de diciembre de 2019, fue notificado a la Organización Mundial de la Salud (OMS) por primera vez por Wuhan (China), el brote de enfermedad por coronavirus (COVID-19). Sin embargo, no es hasta el mes de marzo, que dicha organización declara el COVID-19 como una pandemia, y que además como primera vez en la historia, proviene de un coronavirus.

Como señala De la Corte (2020), como resultado de la propagación del COVID-19, se ha producido la más grave crisis sanitaria mundial conocida en el último siglo $\mathrm{y}$, aunque los factores que han contribuido a ella son diversos, la tendencia a subestimar los riesgos creados por este proceso ha sido una de las principales causas de esta situación tan crítica. Pero además de la falta de previsión y la subestimación de la amenaza, tanto de los actores políticos, como de expertos y la sociedad en general, señala otros factores de gran interés como son: las características del propio virus, la cantidad y calidad de información disponible sobre las características y evolución de este, la manera errática en que algunos países reaccionaron inicialmente ante la amenaza y cómo se ha construido la percepción del riesgo en la sociedad.

Teniendo en cuenta este interesante análisis del autor De la Corte (2020), parece obvio que antes de que finalice esta crisis sanitaria y socioeconómi-

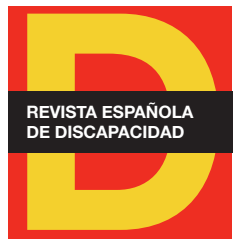

Para citar:

Ortega, E. (2020). "Retos de los stakeholders del ámbito de la discapacidad en materia de empleo tras la pandemia de COVID-19". Revista Española de Discapacidad, 8(l), pp. 209-217.

Doi: <https://doi.org/10.5569/23405104.08.01.12> 
ca se analice y se prevea el posible escenario del empleo de las personas con discapacidad, para evitar caer de nuevo en el error de la falta de previsión y subestimación de la grave situación de fragilidad que acecha a las personas más vulnerables de nuestro entorno.

Como bien destaca el Secretario General de las Naciones Unidas, Antonio Guterres (2020a): "El COVID-19 sigue devastando vidas y medios de subsistencia en todo el mundo, golpeando con más fuerza a los más vulnerables". En esta línea señala que estas personas encaran tres crisis que se combinan en una: en primer lugar, una de carácter sanitario, otra de índole socioeconómica y otra de protección social. Respecto a las personas con discapacidad, hace una reflexión en particular, señalando que estas tienen mayor probabilidad de vivir en la pobreza y que sufren tasas mayores de violencia, abandono y abuso. Otro tema al que también alude y no podemos obviar, es que esta pandemia ha exacerbado la xenofobia, el racismo y la estigmatización (Naciones Unidas 2020a).

En esta línea también encontramos el interesante avance de resultados que ha realizado el Colegio Profesional de Politólogos y Sociólogos de la Comunidad de Madrid (ColPolSoc), sobre un estudio que analiza la crisis del coronavirus, la gestión y consecuencias en términos políticos y sociales, realizado con la opinión de 917 personas expertas de la Ciencia Política y la Sociología, en el mes de mayo. Entre otros datos de interés cabe destacar, respecto al fenómeno de la estigmatización de determinados colectivos, que las personas expertas encuestadas consideran que es uno de los efectos sociales más negativos de esta crisis y que entre otros grupos sociales afecta a las personas en situación de dependencia y/o discapacidad (ColPolSoc, 2020).

\section{Figura 1. Colectivos afectados por la estigmatización como consecuencia del COVID-19}

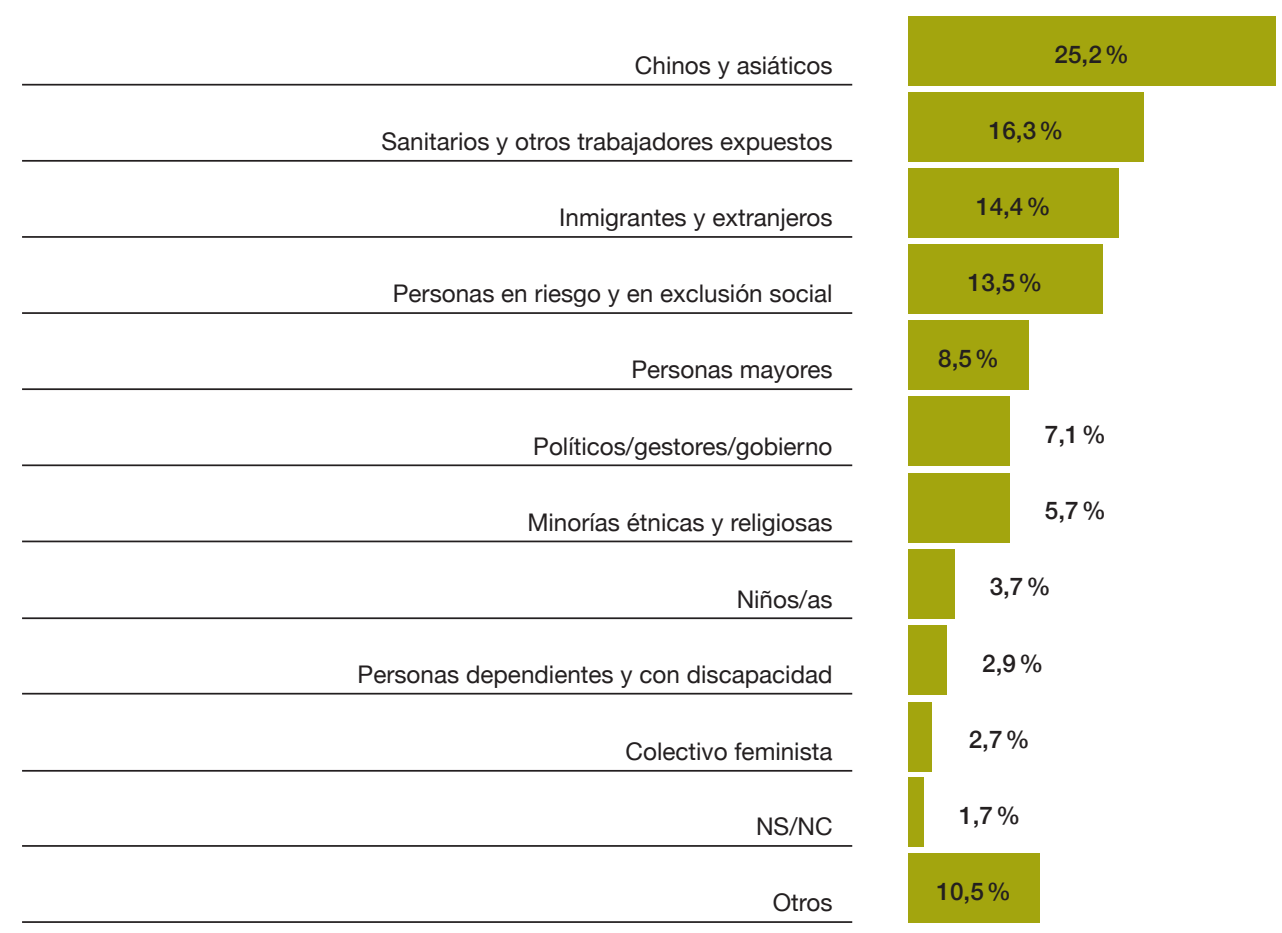

Base: 519 respuestas y porcentaje sobre 828 menciones. Fuente: ColPolSoc, 2020. 
La socióloga Salido (2020) pone de manifiesto que "es casi imposible recordar otro fenómeno de amplitud e intensidad semejantes a nivel global y con una capacidad tan destructiva hasta donde alcanza nuestra memoria" como esta pandemia, y señala: "El mundo, tal como lo conocemos, no volverá a ser el mismo", "la seguridad se ha transformado en inseguridad, la certeza en incertidumbre". También aclama una mirada en particular sobre "niños, ancianos, mujeres que sufren violencia de género, personas con problemas de salud o con necesidades especiales, población en circunstancias de exclusión social...", ya que "la distancia física social puede convertirse en aislamiento" y agravar sus problemas.

\section{Una aproximación a la situación de vulnerabilidad de las personas con discapacidad en España}

El pasado mes, el Secretario General de las Naciones Unidas, Antonio Guterres en su artículo "Una oportunidad única de construir sociedades inclusivas y accesibles" (Guterres, 2020c) y en el documento emitido por Naciones Unidas (2020c) "Informe de políticas: una respuesta a la COVID-19 inclusiva de la discapacidad", se destaca que la pandemia está afectando a los 1000 millones de personas con discapacidad que hay en el mundo. En circunstancias normales, tienen menos probabilidades de acceder a la educación y a la atención de la salud, y menos oportunidades de obtener ingresos o de participar en la comunidad, pero con esta pandemia se está intensificando y creando nuevas amenazas. Opina que es fundamental "velar por que las personas con discapacidad disfruten de los mismos derechos que las demás personas a acceder a la atención sanitaria y a los procedimientos vitales durante la pandemia".

Haciendo una breve reseña de la situación de la discapacidad en España, y teniendo en cuenta los datos facilitados por la Encuesta de Discapacidad, Autonomía Personal y Situaciones de Dependencia (INE, 2008), cabe recordar que en España se estima que el $9 \%$ de la población española tiene alguna discapacidad, suponiendo en torno a 4,12 millones de personas. Es compartido por múltiples autores, que las personas con discapacidad son un grupo en situación de vulnerabilidad social, incluso autores como Díaz (2017) llegan a afirmar que la "falta de igualdad de oportunidades para las personas con discapacidad persiste en muchos ámbitos y supone que el acceso y disfrute de los derechos de ciudadanía sean un $30 \%$ inferior para este colectivo en España". El autor opina que existen múltiples situaciones que favorecen esta situación de desventaja social, como son entre otras la falta de un modelo de educación inclusiva, que favorezca la igualdad educativa en todos sus niveles y altas tasas de desempleo, precariedad laboral y brechas salariales.

Teniendo como base el Informe Olivenza (Jiménez, 2019) del Observatorio Estatal de la Discapacidad, publicado recientemente, caben destacar algunos datos que evidencian la situación de vulnerabilidad y riesgo de exclusión social que comparten las personas con discapacidad. En este sentido, el informe asevera que el $54,6 \%$ de estas personas están en situación de riesgo de pobreza, incluso el 6,2\% de pobreza severa, porcentajes elevados, respecto a la media de la población sin discapacidad¹. En esta línea, el Observatorio

1. Según la Estrategia Europa 2020 se consideran personas en riesgo de pobreza y/o exclusión social (At Risk of Poverty and/or Exclusión, AROPE) a la población que se encuentra en alguna de las tres situaciones que se definen a continuación: personas que viven con bajos ingresos (60 \% de la mediana del ingreso equivalente o por unidad de consumo en el año anterior a la entrevista), y/o personas que sufren privación material severa (al menos 4 de los 9 ítems definidos para medir la privación material) y/o personas que viven en hogares con una intensidad de empleo muy baja (por debajo del $20 \%$ del total de su potencial de trabajo en el año anterior a la entrevista). Informe Olivenza (2019). 
Estatal de la Discapacidad (2020), en el informe emitido sobre la protección a las personas con discapacidad frente a la crisis del COVID-19, ha puesto el foco en explicar cómo estas desigualdades se incrementan en situaciones de crisis.

Respecto a la situación del empleo de las personas con discapacidad, según el Informe 5 ODISMET (2020) elaborado por Fundación ONCE, en España hay 1.899 .800 personas con discapacidad en edad laboral (entre 16 y 64 años), y conforman el 6,3\% de la fuerza productiva de nuestro país. Sin embargo, tan sólo el $1,6 \%$ de todos los contratos que se realizan en el mercado laboral español son a personas con discapacidad, y de estos, el $24,6 \%$ son realizados por centros especiales de empleo.

Este informe ODISMET (2020), realiza un profundo análisis del mercado de trabajo y el nivel de integración sociolaboral de las personas con discapacidad y, entre las principales conclusiones, resalta que existe una situación estructural de desventaja social en el acceso al empleo de las personas con discapacidad. Actualmente, sigue existiendo una gran diferencia respecto a la población general, ya que como se puede observar en dicho informe, tan sólo el $25,8 \%$ de las personas en edad laboral que tienen discapacidad está trabajando, frente al $65,9 \%$ de la población general. Otros datos relevantes son la diferencia salarial existente, que ya se mencionó anteriormente, que ronda los cerca de 4.000 euros anuales y la precariedad laboral. Respecto a este tema, se observa que la rotación laboral en 2018 ha alcanzado su porcentaje más alto. Agravando aún más la situación, se detecta que los grupos más vulnerables dentro de las personas con discapacidad, como son mujeres, jóvenes o tipos específicos de discapacidad, acusan una situación de mayor vulnerabilidad.

Por tanto, la baja tasa de empleo, la precariedad laboral y la falta de protección social del $33,2 \%$ de las personas con discapacidad que no tienen acceso a prestaciones económicas como pensiones contributivas y no contributivas (ODISMET, 2020), ratifica la extrema situación de vulnerabilidad en la que se encontraba un porcentaje muy importante de personas con discapacidad, antes de la pandemia por COVID-19. En esta línea además encontramos que según los diferentes informes que analizan los indicadores AROPE, como son los realizados por la Red Europea de Lucha contra la Pobreza y la Exclusión Social (EAPN, 2018), el empleo no evita el riesgo de pobreza en España.

\section{Crisis económica y laboral provocada por la pandemia del COVID-19: retos para los stakeholders del ámbito de la discapacidad}

La pandemia provocada por COVID-19 no puede ser entendida fuera del marco y concepto de la globalización. Esta ha conllevado la propagación sin límites y fronteras del coronavirus, una gran crisis mundial sanitaria y social y, como no podemos obviar, una de las grandes crisis económicas y laborales de la historia. Según la Organización Internacional del Trabajo (ILO, 2020), la crisis económica y laboral provocada por la pandemia del COVID-19 podría aumentar el desempleo mundial en casi 25 millones de personas. Sin embargo, señalan que, si se produce una respuesta política coordinada a nivel internacional, el impacto sobre el desempleo mundial sería menor. Las medidas urgentes que reclaman giran en torno a tres ejes funda- 
mentales: proteger a los trabajadores en el lugar de trabajo; estimular la economía y el empleo, y sostener los puestos de trabajo y los ingresos.

Teniendo en cuenta esta propuesta de la ILO (2020), a continuación se analizará la situación de las personas con discapacidad como grupo en situación especial de vulnerabilidad social y las posibles medidas que se podrían desarrollar para intentar frenar el impacto. Porque como ha urgido António Guterres (2020b, 2020c), Secretario General de la ONU y el informe de Naciones Unidas (2020b): Shared responsibility, global solidarity: Responding to the socio-economic impacts of COVID-19, los gobiernos deben tener en cuenta que "las personas con discapacidad deben estar en el centro de la respuesta al COVID-19 y en los esfuerzos de recuperación".

\subsection{Adaptación al mercado laboral 2.0 y desarrollo de nuevas competencias profesionales}

El mercado laboral 2.0 o como algunos autores denominan empleabilidad 2.0 (Gómez Grass et al., 2011), se caracteriza a grandes rasgos por la automatización de los procesos productivos por la consolidación de la inteligencia artificial; reducción de trabajos no cualificados derivados de dicha automatización; nuevos modelos de trabajo (teletrabajo, productividad por objetivos, flexibilidad laboral, etc.) y el desarrollo de nuevas actividades profesionales que, como aseguran diferentes autores, incluso a día de hoy son desconocidas.

Ante este escenario, los retos a afrontar por los diferentes agentes sociales como son administración pública, las empresas, las personas con discapacidad, el movimiento organizado de la discapacidad y los centros especiales de empleo, como instrumento de inclusión en el empleo para un porcentaje importante de personas con discapacidad, son los siguientes:

- $\quad$ Analizar y estar preparados para ocupar y/o liderar nuevos nichos de empleo.

- La formación y capacitación profesional de las personas con discapacidad. En esta línea, cabe destacar varios puntos específicos:

- Formación en actividades profesionales emergentes.

- Desarrollo de las competencias profesionales más demandadas (capacidad de innovar, analítica, rigor y alta calidad; habilidades directivas y de liderazgo; oratoria y alta interacción social; trabajo en equipo, versatilidad y adaptabilidad a los cambios; etc.).

- Metodología educativa adaptada a la Era 2.0 y accesible para todas las personas, independientemente de las necesidades educativas que presenten.

- Apuesta por la formación dual y la formación profesional. Actualmente según diversos informes existe una sobrecualificación del mercado laboral español, y no adaptada a las nuevas necesidades del mercado.

- Las tecnologías de la información y la comunicación (TIC), la inteligencia artificial y la robótica como eje central de la actividad laboral. Desde todos los ámbitos y actividades profesionales hay que analizar y desarrollar su posible impacto o vertiente en las TIC. A modo de ejemplo, si tu actividad profesional es la abogacía, especializarte en temas relacionados con estas (ciberseguridad, protección de datos, etc.); si eres sociólogo, centrarte en el análisis de Big Data; si tienes un centro especial de empleo, dedicarte a la digitalización documental, etc. 
- Adaptarse y consolidar los nuevos modelos de trabajo, como es el trabajo por objetivos, la flexibilidad laboral o el teletrabajo. En este sentido se presentan los siguientes retos:

- Formación en TIC para poder utilizar estas herramientas como instrumentos fundamentales de trabajo.

- Accesibilidad y adaptación de los puestos de trabajo para poder desarrollar el teletrabajo (plataformas de trabajo accesibles).

- Universalización de las TIC e Internet. Todavía en España, sobre todo en las zonas rurales, existe un porcentaje importante de personas que no tienen acceso a estos instrumentos fundamentales.

\subsection{Fortalecimiento del estado de bienestar social y legitimación de su sistema de protección}

A pesar de que la tendencia actual es el debilitamiento del estado de bienestar, se aboga por la defensa de este y la legitimación de su sistema de protección social. No obstante, es importante adaptarse a la nueva realidad social e introducir cambios de gran calado:

- Cobertura de las necesidades de todas las personas teniendo en cuenta de forma integral la Convención sobre los Derechos de las Personas con Discapacidad (2006). Actualmente el sistema de protección social tiene un carácter puramente asistencial y no cubre de forma integral todas las necesidades que presenta la persona según su proyecto de vida. Por otro lado, se centra en la pobreza o situaciones de vulnerabilidad extrema, obviando las necesidades y situaciones de las personas que están en una situación moderada o en el umbral de la situación de exclusión. Es fundamental la universalización del estado de bienestar.

- Avanzar en la desmercantilización social, donde el empleo es el eje central de la inclusión social. Un número importante de personas con discapacidad va a presentar grandes dificultades para reconvertirse al nuevo mercado laboral, por ello es fundamental que el sistema de protección social tenga la capacidad de afrontar esta situación manteniendo la calidad de vida de todas las personas. En esta línea las rentas mínimas garantizadas juegan un papel crucial.

- Compatibilidad y flexibilidad entre el mercado laboral y el sistema de protección social. En este sentido, cabe resaltar que es fundamental que el sistema de protección social pueda complementar las nuevas necesidades que genera el subempleo en España (bajos salarios y jornadas laborales reducidas y discontinuas), y en especial a las personas con discapacidad, ya que esta genera un sobrecoste económico en su vida cotidiana. Del mismo modo, es fundamental que, además de la compatibilidad entre prestaciones que complemente la precariedad laboral existente, exista flexibilidad y agilidad para pasar de un sistema a otro. Es decir, que una persona que tenga reconocida una prestación por gran invalidez pueda acceder al empleo en un periodo de su vida si lo decide, y que pueda volver de nuevo a la prestación en caso de necesidad, sin ninguna repercusión negativa como la pérdida de derechos previos adquiridos.

- Protección de los derechos de los trabajadores con discapacidad. Recientemente hemos sido testigos de la tentativa de penalizar y vulnerar los derechos de los trabajadores por presentar una situación de enfermedad prolongada. En esta línea, las personas con discapacidad se encuentran en una situación especial de vulnerabilidad, por ello, es fundamental blindar los derechos de los trabajadores con discapacidad. 
- Del mismo modo hay que ofrecer especial atención a las personas con discapacidad que aúnan múltiples variables que aumentan su situación de vulnerabilidad social ante el empleo como son las mujeres, los jóvenes, mayores o parados de larga duración, entre otros.

3.3. Medidas adicionales de protección social para el sector empresarial vinculado al ámbito de la discapacidad: los centros especiales de empleo (CEE)

- Estas medidas hacen referencia a todas aquellas que incluyen la protección social y el apoyo para que los CEE puedan mantener el empleo de todos los trabajadores con discapacidad y con unas condiciones adecuadas (evitar reducciones de jornada no consensuadas o solicitadas por el trabajador, es decir, el trabajo a jornada reducida, salarios adecuados, etc.) y la concesión de ayudas financieras y desgravaciones fiscales.

- La Ley 9/2017, de 8 de noviembre, de Contratos del Sector Público, por la que se transponen al ordenamiento jurídico español las Directivas del Parlamento Europeo y del Consejo 2014/23/UE y 2014/24/ UE, de 26 de febrero de 2014 (EDL 2017/226876), profundiza en la vertiente social de la contratación pública, y uno de los objetivos de la reforma consiste en introducir nuevas consideraciones en la contratación pública que permitan a los órganos de contratación dar prioridad a la calidad, consideraciones medioambientales, aspectos sociales o a la innovación. Es fundamental impulsar y velar por el cumplimiento de esta ley como estímulo económico y de empleo de los CEE.

\subsection{La protección de los trabajadores en el lugar de trabajo}

A pesar de concluir el estado de alarma, la pandemia de COVID-19 no acabará en una fecha concreta y delimitada. Por ello, es fundamental que tras salir de la situación de crisis se vele por la protección de los trabajadores con discapacidad y sus familias en el entorno laboral, ya que son colectivos en situación de mayor vulnerabilidad a los efectos directos del coronavirus. En esta línea y en consonancia con las recomendaciones y directivas de la OMS y la ILO (2020), hay que hacer hincapié en los aspectos siguientes: Mejora de las medidas de seguridad en el trabajo (distanciamiento social, suministro de equipos de protección, procedimientos de higiene y métodos de organización del trabajo, etc.).

- Fomento de acuerdos laborales flexibles y adecuados como, por ejemplo, el trabajo a distancia.

- Prevención de la discriminación y la exclusión con respecto al COVID-19.

- Fomento del acceso universal a servicios sanitarios, en particular para trabajadores no asegurados y sus familias.

- Ampliación del derecho a bajas remuneradas o subsidios por enfermedad, para ocuparse del cuidado de los hijos o atender a otras personas, en el marco de programas de financiación conjunta, a fin de garantizar la estabilidad de los ingresos de personas enfermas, en situación de cuarentena o responsables del cuidado de niños, personas de edad avanzada, con discapacidad u otros miembros de su familia. 


\section{Referencias bibliográficas}

Colegio Profesional de Politólogos y Sociólogos de la Comunidad de Madrid (2020). Epidemia y sociedad: profesionales de la ciencia política y la sociología ante el COVID-19. Recuperado de https://www.colpolsoc.org/ documents/panel/entradas/Documento_Noticia_231.pdf.

De la Corte, I. (2020). ¿Por qué se subestimó al Covid-19? Un análisis preliminar desde la Psicología y la Sociología del Riesgo. Recuperado de https://global-strategy.org/por-que-se-subestimo-al-covid-19-un-analisis-preliminar-desde-la-psicologia-y-la-sociologia-del-riesgo/.

Díaz, E. (2017). El acceso a la condición de ciudadanía de las personas con discapacidad en España. Un estudio sobre la desigualdad por razón de discapacidad. Madrid: Cermi - Ediciones Cinca.

EAPN. Red Europea de Lucha contra la Pobreza y la Exclusión Social (2018). El estado de la pobreza: seguimiento del indicador de pobreza y exclusión social en España 2008-2017. Recuperado de https://www.eapn.es/estadodepobreza/ARCHIVO/documentos/Informe_AROPE_2018.pdf.

España. Ley 9/2017, de 8 de noviembre, de contratos del sector público, por la que se transponen al ordenamiento jurídico español las Directivas del Parlamento Europeo y del Consejo 2014/23/UE y 2014/24/UE. Boletín Oficial del Estado, 26 de febrero de 2014, núm. 272.

Gómez Grass, J.M., et al. (2011). Empleabilidad 2.0. III Guía de orientación universitaria para el empleo. Recuperado de https://observatorio.umh.es/files/2011/06/2011-empleabilidad-20.pdf.

Guterres, A. (2020a). El COVID-19 es una oportunidad para redefinir los desplazamientos humanos. Recuperado de https://www.un.org/es/coronavirus/articles/efectos-covid-19-en-refugiados-desplazados-migrantes.

Guterres, A. (2020b). La recuperación de la crisis de la COVID-19 deberá conducirnos a una economía diferente. Recuperado de https://www.un.org/es/coronavirus/articles/launch-report-socio-economic-impacts-covid-19.

Guterres, A. (2020c). Una oportunidad única de construir sociedades inclusivas y accesibles. Recuperado de https://www.un.org/es/coronavirus/articles/impacto_covid-19_personas_con_discapacidad.

ILO. International Labour Organization (2020). COVID-19 and the world of work: Impact and policy responses. Recuperado de https://www.ilo.org/wcmsp5/groups/public/---dgreports/---dcomm/documents/briefingnote/ wcms_738753.pdf.

INE (2008). Encuesta de Discapacidad, Autonomía Personal y Situaciones de Dependencia. Madrid: Instituto Nacional de Estadística.

Jiménez, A. (2019). Informe Olivenza 2019, sobre la situación general de la discapacidad en España. Madrid: Observatorio Estatal de la Discapacidad.

Naciones Unidas (2020a). Policy brief: COVID-19 and people on the move. Recuperado de https://www.un.org/ sites/un2.un.org/files/sg_policy_brief_on_people_on_the_move.pdf

Naciones Unidas (2020b). Shared responsibility, global solidarity: Responding to the socio-economic impacts of COVID-19. Recuperado de https://www.un.org/sites/un2.un.org/files/sg_report_socio-economic_impact_of_covid19.pdf

Naciones Unidas (2020c). Informe de políticas: Una respuesta a la COVID-19 inclusiva de la discapacidad. Recuperado de https://www.un.org/sites/un2.un.org/files/spanish_disability_brief.pdf 
Observatorio Estatal de la Discapacidad (2020). Proteger a las personas con discapacidad frente a la crisis de la COVID-19. Recuperado de https://www.cermi.es/sites/default/files/docs/novedades/NOTA\%20OED\%20 PCD\%20CRISIS\%20COVID19\%5B25206\%5D.pdf.

ODISMET. Observatorio sobre Discapacidad y Mercado de Trabajo en España. (2020). La situación de las personas con discapacidad en el mercado laboral. Informe general. Principales resultados. Madrid: Fundación Once.

Salido O. (2020). Reflexiones COVID-19: la mirada de las facultades. Ciencias Políticas y Sociología. Departamento de Sociología Aplicada. Recuperado de https://www.ucm.es/tribunacomplutense/revcul/tribunacomplutense/ doc25013.pdf. 